Hautarzt $2020 \cdot 71: 506-510$

https://doi.org/10.1007/s00105-020-04596-1

Online publiziert: 28. April 2020

(c) Der/die Autor(en) 2020

\title{
G. Schneider
}

Sektion für Psychosomatische Medizin und Psychotherapie, Klinik für Psychische Gesundheit, Münster, Deutschland

\section{Diagnostik von psychischen Faktoren bei chronischem Pruritus}

ßern [10, 27]. Daraus können sich klinisch relevante psychische Störungen wie Anpassungsstörungen, Angst- oder depressive Störungen, teils sogar mit Suizidalität, entwickeln [2]. mnese oder auch durch den Einsatz psychometrischer Instrumente erfolgen. gen können folgende 2 Screeningfragen gestellt werden - mit einer Sensitivität von $96 \%$ und einer Spezifität von $57 \%$ ein sehr zeitökonomisches Vorgehen [4, 28]:

- Fühlten Sie sich im letzten Monat

- Hatten Sie im letzten Monat deutlich

Für die Diagnose einer Panikstörung oder einer generalisierten Angststörung können orientierend folgende Fragen gestellt werden [4]:

- Hatten Sie schon einmal einen Angst-

- Haben Sie sich schon einmal über nischer Pruritus zu erheblichem subjektivem Leiden der Betroffenen führen kann: Dies kann sich in Einschränkungen der Lebensqualität, Schlafstörungen, Störungen der Sexualität, Stimmungsverschlechterung mit Angst und/ oder Depressivität, Belastung zwischenmenschlicher Beziehungen, Erleben von Stigmatisierung, sozialem Rückzug äu-
Die Diagnostik kann durch die Ana-

Für die Diagnose depressiver Störunhäufig niedergeschlagen, traurig, bedrückt oder hoffnungslos? weniger Lust und Freude an Dingen, die Sie sonst gerne tun? anfall, bei dem Sie ganz plötzlich von starker Angst, Beklommenheit oder Unruhe überfallen wurden? mindestens einen Monat oder länger ängstlich, angespannt und voll ängstlicher Besorgnis gefühlt?

Einschränkungen der Lebensqualität, Angst und Depressivität können auch mittels validierter und reliabler psychometrischer Instrumente erfasst werden. Die Langfassung der S2k-Leitlinie zur Diagnostik und Therapie des chronischen Pruritus [23] empfiehlt die in - Tab. 2 aufgeführten Fragebögen.

Geschlechtsspezifische Unterschiede wurden insoweit gezeigt, dass Frauen höhere Pruritusintensitäten angeben als Männer sowie eine höhere Ausprägung von Ängstlichkeit und Depressivität [22, 24].

\section{B. Psychische Aspekte in Entstehung und Verlauf eines chronischen Pruritus}

\section{B1. Klinisch relevante psychische/ psychosomatische Einflussfaktoren}

Wenn für Auslösung und/oder Verlauf eines CP neben dem gleichzeitigen Vorliegen einer oder mehrerer organischer Pruritusursachen relevante psychische/ psychosomatische Einflussfaktoren $\mathrm{zu}$ identifizieren sind, wird empfohlen, diese nach ICD(Internationale Statistische Klassifikation der Krankheiten und verwandter Gesundheitsprobleme)-10 als „F54: Psychologische und Verhaltensfaktoren bei andernorts klassifizierten Erkrankungen“ zu klassifizieren.

Aus der Literatur sind vielfältige EinWenn die Kriterien einer depressiven oder Angststörung nach ICD-10 nicht erfüllt sind, die Belastung des Patienten aber klinisch relevant und Folge des CP ist, kann die Diagnose einer Anpassungsstörung im Sinne von Problemen der Symptombewältigung zutreffen. flussfaktoren auf Auslösung und Verlauf von Pruritus bekannt: Pruritus und Kratzen können durch (audio)visuelle Reize induziert werden [17]. Positive und negative Erwartungen, die auch durch entsprechende Instruktionen induziert wer- 
Tab. 1 Systematik Pruritus-assoziierter psychischer Störungen

A. Psychische Beeinträchtigungen/Störungen als Reaktion auf chronischen Pruritus: z. B. Anpassungsstörungen [ICD-10: F43], depressive Störungen [ICD-10: F32-F33], Angststörungen [ICD-10: F40-F41]

B. Psychische Aspekte in Entstehung und Verlauf eines chronischen Pruritus

- B1. Klinisch relevante psychische/psychosomatische Einflussfaktoren, die Auslösung und Verlauf jedoch nicht alleine bzw. überwiegend erklären: psychologische und Verhaltensfaktoren bei andernorts klassifizierten Erkrankungen [ICD-10: F54]

- B2. Somatoformer Pruritus [ICD-10: F45.0, F45.1, F45.8]

- B3. Pruritus bei coenästhetischer Schizophrenie [ICD-10: F 20]

- B4. Selbst induzierte Kratzartefakte mit oder ohne Pruritus bei abnormen Gewohnheiten und Störungen der Impulskontrolle [ICD-10: F63.3], Artefaktstörungen [ICD-10: F68.1], Zwangsstörungen [F42.1]

C. Komorbidität mit prinzipiell jeder sonstigen psychischen/psychosomatischen Störung möglich, die ihrerseits das Management des Pruritus erschweren und den Krankheitsverlauf beeinflussen kann (z. B. durch Complianceprobleme bei Persönlichkeitsstörungen, Substanzmissbrauch, organischen oder schizophrenen Psychosen u. a.); bei Relevanz für den Verlauf unter ICD-10: F54 klassifizierbar

ICD Internationale Statistische Klassifikation der Krankheiten und verwandter Gesundheitsprobleme

Tab. 2 Psychometrische Instrumente zurErfassung von Lebensqualität, Angst und Depressivität bei chronischem Pruritus

\begin{tabular}{|ll}
\hline Lebensqualität & $\begin{array}{l}\text { Dermatology Life Quality Index (DLQI) bei Dermatosen } \\
\text { ItchyQol }\end{array}$ \\
Depressivität & $\begin{array}{l}\text { Hospital Anxiety and Depression Scale (HADS) } \\
\text { Patient Health Questionnaire (PHQ) }\end{array}$ \\
\hline Ängstlichkeit & $\begin{array}{l}\text { Beck's Depressions-Inventar (BDI) } \\
\text { Hospital Anxiety and Depression Scale (HADS) }\end{array}$ \\
& Patient Health Questionnaire (PHQ)
\end{tabular}

den können (im Sinne von Placebo- oder Noceboeffekten), beeinflussen die Stärke der Prurituswahrnehmung, das Kratzverhalten, die physiologischen Hautreaktionen nach experimentell induziertem Pruritus $[25,26]$.

\section{》) Pruritus und Kratzen können durch psychische Faktoren beeinflusst werden}

Ängstlichkeit [9], Depressivität [16] sowie bestimmte Persönlichkeitseigenschaften wie hoher Neurotizismus (im Sinne emotionaler Instabilität mit Tendenz, mehr negative Emotionen zu erleben), geringe Verträglichkeit $[16,19$, 20] und niedriges Selbstwirksamkeitserleben [1] waren mit einer stärkeren Ausprägung der Pruritusempfindung und des Kratzens assoziiert. Resilienz (psychische Widerstandsfähigkeit) und Neurotizismus zeigten Zusammenhänge zu einem stärkeren Ansprechen auf Placeboinstruktionen für Pruritus [3].

Andere Studien zeigten Assoziationen zwischen subjektivem Stress, belastenden Lebensereignissen und Ausprägung der jeweiligen Hautsymptomatik und Stärke der Pruritusempfindung bei Hauterkrankungen (u. a. [8]). Bei Gesunden wurden ebenfalls Zusammenhänge zwischen der Häufigkeit von "major life events" in den letzten 6 Monaten bzw. subjektiv wahrgenommenem Stress und der Angabe von Pruritus gezeigt $[18,29]$. Patienten mit CP gaben vor einem sozialen Stresstest höhere Anspannung an und zeigten ausgeprägtere physiologische Stressreaktionen als Gesunde [14].

Kognitive und Verhaltensaspekte können ebenfalls relevanten Einfluss auf die CP-Wahrnehmung und -Bewältigung und seine Behandlung haben.

Das Erleben von Stigmatisierung infolge sichtbarer Hautveränderungen wie Kratzläsionen kann zu sozialem Vermeidungsverhalten und Rückzug führen, was dann wieder durch Wegfallen angenehmer Aktivitäten zu einer zunehmenden Fokussierung auf den Pruritus und darüber zu einer verstärkten Prurituswahrnehmung führen kann.

Negative Kognitionen wie verallgemeinernde und katastrophisierende Gedanken können den Stress durch den Pruritus noch verstärken und zu vermehrtem Pruritus beitragen. Eskonnte gezeigt werden, dass der Zusammenhang von Stress und Pruritus bei atopischer Dermatitis über negative pruritusbezogene Kognitionen vermittelt wird [21].

Der häufig kaum unterdrückbare Kratzimpuls kann zu Spannungen mit nahestehenden Personen führen, die den Betroffenen auffordern, das Kratzen zu unterlassen. Die Kratzimpulse können als nicht unterdrückbar erlebt werden. Teils werden dazu auch Hilfsgegenstände genutzt, oder es entsteht ein automatisiertes Kratzverhalten, bei dem die Betroffenen auch kratzen, wenn es nicht juckt, teils auch ohne dass es ihnen bewusst wird. So entsteht ein Teufelskreis aus Jucken und Kratzen, in dem die Betroffenen kurzfristig durch das Kratzen Erleichterung des Pruritus empfinden, längerfristig jedoch ihre Haut schädigen, was den Pruritus verstärken kann, zu vermehrtem Kratzen führt usw.

Die Diagnostik psychischer Einflussfaktoren in der Hautarzt/Facharztpraxis setzt eine gründliche (und leider meist auch zeitintensive) Anamnese voraus: Einige diagnostische Erfassungsmöglichkeiten und beispielhafte Fragen für die in diesem Abschnitt beschriebenen Aspekte sind in $\bullet$ Tab. 3 aufgeführt:

\section{B2. Somatoformer Pruritus}

Psychische und psychosomatische Faktoren können auch als alleinige oder überwiegende Ursache eines CP infrage kommen, der dann bei Nachweis dieser und Ausschluss relevanter organischer Ursachen als somatoformer Pruritus (ICD10: F45.8 "Sonstige somatoforme Störungen“) klassifiziert wird. Der Begriff „psychogener Pruritus"sollte nicht verwendet 
werden, da dieser keinem anerkannten Klassifikationssystem entspricht.

Somatoforme Störungen sind charakterisiert durch wiederholtes Darbieten körperlicher Symptome, z. B. Pruritus oder Hautbrennen, in Verbindung mit hartnäckigen Forderungen nach medizinischen Untersuchungen trotz wiederholt negativer Ergebnisse und der Versicherung der Ärzte, dass die Symptome nicht körperlich begründbar sind. Gleichzeitig liegen psychosoziale Belastungen vor, die die Symptomatik bedingen und unterhalten können. Die Störung kann monosymptomatisch (nur Pruritus) sein oder polysymptomatisch (Pruritus begleitet von anderen organisch nicht ausreichend erklärbaren körperlichen Beschwerden).

Demgemäß basiert die Diagnosestellung auf dem Ausschluss einer organischen Ursache und dem Nachweis psychosozialer Einflussfaktoren auf Auslösung und Verlauf des Pruritus. Letztere können dem im Abschn. B1 Aufgeführten entsprechen, das diesbezügliche diagnostische Vorgehen wäre das Gleiche.

\section{》) CP ist häufig multifaktoriell bedingt, aber psychische Faktoren können die alleinige Ursache sein}

In einer kürzlich publizierten Studie wurden CP-Patienten, bei denen auf der Basis einer psychosomatischen Konsiliaruntersuchung die Diagnose relevanter psychischer/psychosomatischer Einflussfaktoren in Auslösung/Verlauf des CP oder die Diagnose somatoformer Pruritus gestellt worden war, mit CP-Patienten verglichen, die keine anamnestische oder aktuell diagnostizierte psychische Komorbidität hatten. Dabei bejahten die CP-Patienten mit psychosomatischen Einflussfaktoren bzw. somatoformem Pruritus als Trigger ihres Pruritus signifikant häufiger "Anspannung“ und „emotionale Anspannung“. Sie bejahten signifikant häufiger bestimmte emotionale Adjektive, um ihr Prurituserleben zu beschreiben: „grausam“, „qualvoll“, „aufwühlend“, „entsetzlich“ sowie „der Juckreiz macht mich aggressiv“. Da diese

Hautarzt 2020 - 71:506-510 https://doi.org/10.1007/s00105-020-04596-1

(c) Der/die Autor(en) 2020

\section{G. Schneider}

\section{Diagnostik von psychischen Faktoren bei chronischem Pruritus}

\section{Zusammenfassung}

Hintergrund. Pruritus ist ein häufiges und quälendes Symptom in der Bevölkerung sowie in ärztlichen Praxen.

Ziel der Arbeit. Der vorliegende Beitrag gibt einen Überblick über die Systematik psychischer Faktoren in Entstehung bzw. Verlauf des Pruritus sowie Hinweise zu ihrer jeweiligen Diagnostik

Material und Methode. Es handelt sich um eine Überblicksarbeit.

Ergebnisse. Psychische Beeinträchtigungen/Störungen können als Reaktion auf chronischen Pruritus auftreten, z. B. als Anpassungsstörungen, depressive Störungen, Angststörungen. Psychische Faktoren können auch in Entstehung und Verlauf eines chronischen Pruritus eine Rolle spielen, entweder im Zusammenspiel mit organischen Ursachen oder bei deren Fehlen als somatoformer Pruritus oder Pruritus bei coenästhetischer Schizophrenie. Ferner können selbstinduzierte Kratzartefakte mit oder ohne Pruritus im Rahmen von Störungen der Impulskontrolle,
Zwangsstörungen oder Artefaktstörungen auftreten. Auch eine primär unabhängige Komorbidität mit einer sonstigen psychischen oder psychosomatischen Störung kann das Management des Pruritus erschweren und den Krankheitsverlauf beeinflussen. Es werden Hinweise zur Diagnostik der genannten Faktoren gegeben.

Diskussion. Die Diagnostik psychischer Einflussfaktoren und psychischer Aspekte des chronischen Pruritus ist komplex. Das wichtigste diagnostische Werkzeug ist die gründliche Anamneseerhebung auch bezüglich psychischer Aspekte. Zusätzlich können psychometrische Instrumente zum Einsatz kommen, die das ärztliche Gespräch jedoch nicht ersetzen.

\section{Schlüsselwörter}

Psychosomatische Faktoren · Psychische Störungen · Diagnostik · Anamnese . Somatoformer Pruritus

\section{Diagnosis of psychological aspects in chronic pruritus}

\section{Abstract}

Background. Pruritus is a frequent symptom in the general population and in clinics. Objective. This article gives an overview on psychological and psychosomatic aspects of chronic pruritus and tools for their diagnosis. Materials and methods. This is a review. Results. Chronic pruritus can lead to psychological impairment and psychic diseases, e.g. adjustment disorder, depression. Psychological factors can also be important factors in the etiology and course of chronic pruritus, either in combination with somatic aspects or, in the absence of these, as somatoform pruritus or pruritus in coenasthetic schizophrenia. Self-induced scratch lesions with or without pruritus can be symptoms of impulse control disorders, obsessive-compulsive disease or artificial disorders. Furthermore, independent comorbidity with other psychic disorders can influence the management of chronic pruritus. We provide hints/tools for their diagnosis.

Conclusions. The diagnosis of psychological aspects in chronic pruritus is complex. The main diagnostic tool is the thorough taking of the patient history, including psychological aspects. It can be supplemented by standardized questionnaires, but these can not be substitutes.

\section{Keywords}

Psychosomatic aspects · Psychic disorders · Diagnosis $\cdot$ Medical history taking $\cdot$ Somatoform pruritus
Aspekte jedoch nicht exklusiv bei diesen Patienten zutrafen, sondern auch von manchen CP-Patienten ohne psychische Komorbidität bejaht wurden, können sie allenfalls als Hinweise dienen [12].

\section{B3. Pruritus bei coenästhetischer Schizophrenie}

Schizophrene und wahnhafte Störungen können sich durch taktile Halluzinationen als Pruritus oder die wahnhafte Überzeugung, an einer Infektion z.B. mit Parasiten und dadurch bedingtem Pruritus zu leiden, manifestieren. Die 
Tab. 3 Einige Erfassungsmöglichkeiten und beispielhafte Fragen für relevante psychische/psychosomatische Einflussfaktoren, die Auslösung und Verlauf des Pruritus beeinflussen

\begin{tabular}{|c|c|}
\hline Ziel & Mögliche Fragen bzw. Instrumente \\
\hline \multirow[t]{2}{*}{$\begin{array}{l}\text { Zusammenhänge zu } \\
\text { Lebensereignissen/Stress }\end{array}$} & $\begin{array}{l}\text { Gab es um den Zeitpunkt des Juckreizbeginns (bzw. der Verschlimmerung) besondere Ereignisse in Ihrem Leben? } \\
\text { Haben Sie in diesem Zeitraum besondere Belastungen bzw. Stress erlebt? }\end{array}$ \\
\hline & $\begin{array}{l}\text { Bemerken Sie eine Zunahme oder Abnahme Ihres Juckreizes im Zusammenhang mit bestimmten Situationen/ } \\
\text { Gefühlen/Emotionen/Tätigkeiten? }\end{array}$ \\
\hline \multirow{3}{*}{$\begin{array}{l}\text { Identifikation negativer } \\
\text { Erwartungen/Kognitionen/ } \\
\text { katastrophisierender Gedanken }\end{array}$} & Wenn der Juckreiz ausgeprägt ist, welche Gedanken gehen Ihnen durch den Kopf? \\
\hline & Welche Gefühle haben Sie dabei? \\
\hline & $\begin{array}{l}\text { Zur Vertiefung können Symptomtagebücher, die die Ausprägung des Pruritus, die jeweilige Situation sowie damit } \\
\text { verbundene Gedanken und Gefühle erfassen, zum Einsatz kommen. } \\
\text { Zur Identifikation negativer und katastrophisierender Kognitionen steht im deutschsprachigen Raum auch der Juck- } \\
\text { reiz-Kognitions-Fragebogen oder Eppendorfer Juckreizfragebogen zur Verfügung }\end{array}$ \\
\hline \multirow{2}{*}{$\begin{array}{l}\text { Identifikation negativer } \\
\text { Bewältigungsstrategien }\end{array}$} & Was tun Sie, wenn der Juckreiz besonders ausgeprägt ist? \\
\hline & $\begin{array}{l}\text { Haben Sie aufgrund des Juckreizes bestimmte Aktivitäten aufgegeben, die Ihnen früher Freude bereitet haben, } \\
\text { bzw. sich sozial zurückgezogen? }\end{array}$ \\
\hline $\begin{array}{l}\text { Identifikation bestimmter Per- } \\
\text { sönlichkeitsdispositionen sowie } \\
\text { von Complianceproblemen }\end{array}$ & $\begin{array}{l}\text { Diese können sich aus dem Verlauf der Behandlung/der klinischen Beobachtung erschließen } \\
\text { Ferner stehen zur Persönlichkeitsdiagnostik standardisierte psychometrische Instrumente zur Verfügung, die aber in } \\
\text { der Regel eher einer spezialisierten psychologischen Diagnostik vorbehalten sein werden (z. B. Neo-Fünf-Faktoren-In- } \\
\text { ventar) }\end{array}$ \\
\hline
\end{tabular}

Diagnostik bereitet keine besonderen Schwierigkeiten, wenn die Patienten offen von diesen Überzeugungen berichten, evtl. auch Material mitbringen, das sie für Parasiten o. Ä. halten, und an diesen Überzeugungen auch festhalten, wenn man ihnen die negativen Untersuchungsergebnisse mitteilt. Pruritus im Rahmen einer coenästhetischen oder sonstigen Psychose sollte als Symptom einer Schizophrenie (ICD-10: F20) klassifiziert werden. Der Patient sollte in einem solchen Verdachtsfall fachärztlich-psychiatrisch untersucht und ggf. behandelt werden.

\section{B4. Selbst induzierte Kratzartefakte mit oder ohne Pruritus}

Bei den artifiziellen Störungen ist das zentrale Symptom die Vortäuschung, Aggravation und/oder Erzeugung körperlicher oder psychischer Krankheitssymptome, die häufig medizinische Behandlungen zur Folge haben. Die Patienten können auch Pruritus angeben und damit Hautläsionen begründen. Die Diagnostik artifizieller Störungen ist deswegen schwierig, weil die Selbstschädigung der Haut teils bewusstseinsfern geschieht und von den Patienten nicht berichtet oder bei Konfrontation damit in der Regel nicht zugegeben wird. Diagnostische Hinweise sind klinisch ungewöhnliche Läsionen, die trotz Behandlung nicht zuheilen, und
Leugnen der Manipulation durch den $\mathrm{Pa}$ tienten.

Davon abzugrenzen sind „Paraartefakte“, bei denen den Patienten ihre selbstschädigenden Handlungen bewusst sind und sie diese auch zugeben, jedoch nicht beenden können: Die Diagnostik erfolgt durch die typische Anamnese: Exzessives, auch automatisches Kratzen in diesem Sinne („neurotische Exkoriationen“) kann man nach der ICD-10 als Impulskontrollstörung (ICD-10: F63.3) klassifizieren. Bei schweren Zwangsstörungen, insbesondere Zwangshandlungen mit Waschzwang können Austrocknen/Schädigung der Haut, dadurch bedingte Ekzeme und Superinfektionen ebenfalls Pruritus auslösen. Die Diagnostik erfolgt ebenfalls auf Basis der typischen Anamnese, dass Patienten berichten, dass sie sich sehr häufig die Hände waschen müssen, dies zwar als übertrieben oder unsinnig erkennen, aber trotz Versuchen, dem Widerstand zu leisten, nicht einstellen können.

\section{Komorbidität mit psychischen Störungen}

Untersuchungen in der Allgemeinbevölkerung zeigen Lebenszeitprävalenzen von 25-40\% psychischer Störungen [5]. Dies bedeutet, dass bei einem Teil der von Pruritus Betroffenen unabhängig davon gleichzeitig eine psychische Stö- rung vorliegen kann, die ihrerseits die Behandlung des Juckens erschweren und den Krankheitsverlauf beeinflussen kann (z.B. durch Complianceprobleme bei Persönlichkeitsstörungen, Substanzabhängigkeiten, Psychosen u.a.).

Deswegen sollte die Anamnese auch Fragen nach aktuellen oder früheren psychischen oder psychosomatischen Erkrankungen sowie psychiatrischen oder psychotherapeutischen Behandlungen umfassen.

\section{Fazit für die Praxis}

- Psychische Störungen und Einflussfaktoren bei chronischem Pruritus (CP) sind häufig.

- Sie können Ursache oder Folge des CP sein und sein Management erheblich beeinflussen.

- Ihre Diagnostik erfolgt vorwiegend durch die gründliche Anamnese.

- Fragebögen können ergänzend eingesetzt werden.

- Bei klinisch relevanten psychischen Einflussfaktoren kann die Behandlung zunächst im Rahmen der psychosomatischen Grundversorgung erfolgen.

- Bei mangelndem Erfolg sollte der Patient einer weiteren spezialisierten psychischen Diagnostik und ggf. Behandlung zugeführt werden. 


\section{Korrespondenzadresse}

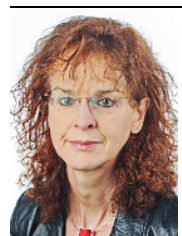

Prof. Dr. G. Schneider

Sektion für Psychosomatische Medizin und Psychotherapie, Klinik für Psychische

Gesundheit

Albert-Schweitzer Campus 1, Gebäude A9, 48149 Münster,

Deutschland

Gudrun.Schneider@

ukmuenster.de

\section{Einhaltung ethischer Richtlinien}

Interessenkonflikt. G. Schneider gibt an, dass kein Interessenkonflikt besteht.

Für diesen Beitrag wurden vom Autor keine Studien an Menschen oder Tieren durchgeführt. Für die aufgeführten Studien gelten die jeweils dort angegebenen ethischen Richtlinien.

Open Access. Dieser Artikel wird unter der Creative Commons Namensnennung 4.0 International Lizenz veröffentlicht, welche die Nutzung, Vervielfältigung, Bearbeitung, Verbreitung und Wiedergabe in jeglichem Medium und Format erlaubt, sofern Sie den/die ursprünglichen Autor(en) und die Quelle ordnungsgemäß nennen, einen Link zur Creative Commons Lizenz beifügen und angeben, ob Änderungen vorgenommen wurden.

Die in diesem Artikel enthaltenen Bilder und sonstiges Drittmaterial unterliegen ebenfalls der genannten Creative Commons Lizenz, sofern sich aus der Abbildungslegende nichts anderes ergibt. Sofern das betreffende Material nicht unter der genannten Creative Commons Lizenz steht und die betreffende Handlung nicht nach gesetzlichen Vorschriften erlaubt ist, ist für die oben aufgeführten Weiterverwendungen des Materials die Einwilligung des jeweiligen Rechteinhabers einzuholen.

Weitere Details zur Lizenz entnehmen Sie bitte der Lizenzinformation auf http://creativecommons.org/ licenses/by/4.0/deed.de.

\section{Literatur}

1. Dalgard F, Stern R, Lien L, Hauser S (2012) Itch, stress and self-efficacy among 18-year-old boys and girls: a Norwegian population-based crosssectional study. Acta Derm Venereol 92:547-552

2. Dalgard FJ, Svensson $\AA$, Halvorsen JA et al (2019) Itch and mental health in dermatological patients across Europe: a cross sectional study in 13 countries. J Invest Dermatol 140:568-573

3. Darragh M, Booth RJ, Consedine NS (2016) Trait predictors of placebo responses in itch. Psychol Health Med 21:769-775

4. DGPPN, BÄK，KBV, AWMF (Hrsg) (2017) S3Leitlinie/Nationale Versorgungsleitlinie Unipolare Depression - Kurzfassung, 2. Aufl. https://doi.org/ 10.6101/AZQ/000366 (Version 1)

5. Jacobi F, Höfler M, Siegert J et al (2014) Twelvemonth prevalence, comorbidity and correlates of mental disorders in Germany: the mental health module of the German health interview and examination survey for adults (DEGS1-MH). Int J Methods Psychiatr Res 23:304-319

6. Kopyciok ME, Ständer HF, Osada N et al (2016) Prevalence and characteristics of pruritus: a one-week cross-sectional study in a German dermatology practice. Acta Derm Venereol 96:50-55

7. Matterne U, Apfelbacher CJ, Loerbroks A et al (2011) Prevalence, correlates and characteristics of chronic pruritus: a population-based crosssectional study. Acta Derm Venereol 91:674-679

8. Mochizuki H, Lavery MJ, Nattkemper LA et al (2019) Impact of acute stress on itch sensation and scratching behaviour in patients with atopic dermatitis and healthy controls. Br J Dermatol 180:689-690

9. Ogden J, Zoukas S (2009) Generating physical symptoms from visual cues: an experimental study. Psychol Health Med 14:695-704

10. Pereira MP, Ständer S (2017) Assessment of severity and burden of pruritus. Allergol Int 66:3-7

11. Schneider G, Driesch G, Heuft G, Evers S, Luger TA, Ständer S (2006) Psychosomatic cofactors and psychiatric comorbidity in patients with chronic itch.Clin Exp Dermatol 31:762-767

12. Schneider G, Grebe A, Bruland P et al (2020) Criteria suggestive of psychological components of itch and somatoform itch: study of a large sample of patients with chronic pruritus. Acta Derm Venereol. https://doi.org/10.2340/00015555-3424

13. Schneider G, Grebe A, Bruland P, Heuft G, Ständer S (2019) Chronic pruritus patients with psychiatric and psychosomatic comorbidity are highly burdened: a longitudinal study. J Eur Acad Dermatol Venereol 33:e288-e291.https://doi.org/ $10.1111 / j d v .15559$

14. Schneider G, Stumpf A, Burgmer Met al (2018) Are patients with chronic pruritus more susceptible to social stress than healthy controls? An experimental case-control study. Br J Dermatol 179:1174-1176

15. Schofield JK, Fleming D, Grindlay D, Williams H (2011) Skin conditions are the commonest new reason people present to general practitioners in England and Wales. Br J Dermatol 165:1044-1050

16. Schut C, Bosbach S, Gieler U, Kupfer J (2014) Personality traits, depression and itch in patients with atopic dermatitis in an experimental setting: a regressionanalysis. Acta Derm Venereol 94:20-25

17. Schut C, Grossman S, Gieler U et al (2015) Contagious itch: what we know and what we would like to know. Front Hum Neurosci 9:57. https://doi.org/10.3389/fnhum.2015.00057

18. Schut C, Mollanazar NK, Sethi M et al (2016) Psychological stress and skin symptoms in college students: results of a cross-sectional webbased questionnaire study. Acta Derm Venereol 96:550-551

19. SchutC, Muhl S, Reinisch Ket al (2015) Agreeableness and self-consciousness as predictors of induced scratching and itch in patients with psoriasis. Int J Behav Med 22:726-734

20. Schut C, Rädel A, Frey L et al (2016) Role of personality and expectations for itch and scratching induced by audiovisual itch stimuli. Eur JPain 20:14-18

21. Schut C, Weik U, Tews N et al (2015) Coping as mediator of the relationship between stress and itch in patients with atopic dermatitis: a regression and mediation analysis. Exp Dermatol 24:148-150

22. Ständer S, Stumpf A, Osada N et al (2013) Gender differences in chronic pruritus: women present different, morbidity, more scratch lesions and higher burden. Br J Dermatol 168:1273-1280

23. Ständer S, Zeidler C, Augustin M et al (2017) S2k-Leitlinie zur Diagnostik und Therapie des chronischen Pruritus - Update - Kurzversion. JDtsch Dermatol Ges 15:860-873

24. Stumpf A, Ständer S, Warlich Betal (2015) Relation between the characteristics and psychological comorbidities of chronic pruritus differ between men and women: women are more anxious than men. Br J Dermatol 172:1323-1328

25. Stumpf A, Zerey V, Heuft G, Ständer S et al (2016) Itch perception and skin reactions as modulated by verbal suggestions: role of participant's and investigator's sex. Acta Derm Venereol 96:619-623

26. van Laarhoven AIM, van der Sman-Mauriks IM, Donders ART et al (2015) Placebo effects on itch: a meta-analysis of clinical trials of patients with dermatological conditions. J Invest Dermatol 135:1234-1243

27. Warlich B, Fritz F, Osada N et al (2015) Healthrelated quality of life in chronic pruritus: an analysis related to disease etiology, clinical skin conditions and itch intensity. Dermatology 231:253-259

28. Whooley MA, Avins AL, Miranda J, Browner WS (1997) Case-finding instruments for depression. Two questions are as good as many. J Gen Intern Med 12:439-445

29. Yamamoto $Y$, Yamazaki S, Hayashino $Y$ et al (2009) Association between frequency of pruritic symptoms and perceived psychological stress: a Japanese population-based study. Arch Dermato 145:1384-1388 